\title{
Quark Masses and Mixings in the 3-3-1 Model with Neutral Leptons Based on $D_{4}$ Flavor Symmetry
}

\author{
V. V. Vien* \\ Department of Physics, Tay Nguyen University, \\ 567 Le Duan, Buon Ma Thuot, DakLak, Vietnam \\ H. N.Long ${ }^{\dagger}$ \\ Institute of Physics, Vietnam Academy of Science and Technology, \\ 10 Dao Tan, Ba Dinh, Hanoi, Vietnam
}

(Received 6 August 2007)

\begin{abstract}
The $D_{4}$ flavor model based on $\mathrm{SU}(3)_{C} \otimes \mathrm{SU}(3)_{L} \otimes \mathrm{U}(1)_{X}$ gauge symmetry that aims at describing quark mass and mixing is updated. After spontaneous breaking of flavor symmetry, with the constraint on the Higgs vacuum expectation values (VEVs) in the Yukawa couplings, all of quarks have consistent masses, and a realistic quark mixing matrix can be realized at the first order of perturbation theory.
\end{abstract}

PACS numbers: 11.30.Hv, 14.65.-q, 11.30.Er

Keywords: Flavor symmetries, Quarks, discrete symmetries

*Electronic address: wvienk16@gmail.com

${ }^{\dagger}$ Electronic address: hnlong@iop.vast.ac.vn 


\section{INTRODUCTION}

One of the most interesting challenges in particle physics is to determine the origin of quark mixing, described by the unitary Cabibbo-Kobayashi-Maskawa (CKM) matrix [1, 2], which is approximately proportional to the identity. The CKM matrix elements are fundamental parameters of the Standard Model (SM), so their precise determination is important. The CKM matrix has many parametrizations [3] 9]; however, the CKM parametrization [1, 2] and the Wolfenstein one [10] are widely used. Recently, the discrete symmetries have been a useful tool for understanding quark and lepton mixing [11-15]. The elements in the CKM matrix have now been determined with high accuracy. The fit results for the magnitudes of all CKM elements in Ref. 16 imply

$$
\left|U_{\mathrm{CKM}}\right|=\left(\begin{array}{ccc}
0.97425 \pm 0.00022 & 0.2253 \pm 0.0008 & (4.13 \pm 0.49) \times 10^{-3} \\
0.225 \pm 0.008 & 0.986 \pm 0.016 & (41.1 \pm 1.3) \times 10^{-3} \\
(8.4 \pm 0.6) \times 10^{-3} & (40.0 \pm 2.7) \times 10^{-3} & 1.021 \pm 0.032
\end{array}\right)
$$

From Eq. (1), it follows that the quark mixing angles are small and completely different from the lepton mixing ones that have been studied widely by many authors in recent years [17-31 and references therein].

In our previous works [21 23, 25 31], the lepton mass and mixing were studied in detail; however, the realistic quark mixing has not been considered. In Ref.24, we studied the 3-3-1 model with neutral fermions based on the $D_{4}$ group in which the quark mixing matrix is unity at the tree-level and the $1-2$ mixing of the ordinary quarks is obtained if the $D_{4}$ symmetry is violated with $1^{\prime}$; i.e, the 12 and 21 entries of the quark mixing matrix $U_{C K M}$ are non-zero if under $\left[\mathrm{SU}(3)_{L}, \mathrm{U}(1)_{X}, \mathrm{U}(1)_{\mathcal{L}}, \underline{D}_{4}\right]$ symmetries, the tensor products of fields in the quark Yukawa interactions are $\left[1,0,0, \underline{1}^{\prime}\right]$ instead of $[1,0,0, \underline{1}]$ as usual. Our aim in this paper is to construct the 3-3-1 model with neutral leptons based on $D_{4}$ flavor symmetry having a quark mixing pattern in agreement with the most recent data.

The basic feature of the model is that all the quark fields act as different singlets under $D_{4}$, and a new parametrization of quark mixing is proposed at the tree-level. The realistic quark mixing is obtained at the first order of perturbation theory when $D_{4}$ symmetry is violated with $1^{\prime}$. The rest of this work is organized as follows: In Section III, we introduce the necessary Higgs fields responsible for the charged lepton as well as the neutrino mass and mixing. Section โII is devoted to quark mixing. We summarize our results and draw 
conclusions in Section IV] Appendix A presents a brief description of the $D_{4}$ theory.

\section{THE MODEL}

The lepton content of the model is the same as that in Ref. 28. In this work, we will concentrate on the quark sector, where under the $\left[\mathrm{SU}(3)_{L}, \mathrm{U}(1)_{X}, \mathrm{U}(1)_{\mathcal{L}}, \underline{D}_{4}\right]$ symmetries, the left- and the right-handed quark fields transform as follows:

$$
\begin{aligned}
& Q_{3 L}=\left(\begin{array}{lll}
u_{3 L} & d_{3 L} & U_{L}
\end{array}\right)^{T} \sim[3,1 / 3,-1 / 3, \underline{1}], \quad u_{3 R}=[1,2 / 3,0, \underline{1}], \quad d_{3 R}=[1,-1 / 3,0, \underline{1}], \\
& Q_{1 L} \equiv\left(d_{1 L}-u_{1 L} \quad D_{1 L}\right)^{T} \sim\left[3^{*}, 0,1 / 3, \underline{1}^{\prime}\right], \quad u_{1 R}=\left[1,2 / 3,0, \underline{1}^{\prime}\right], \quad d_{1 R}=\left[1,-1 / 3,0, \underline{1}^{\prime}\right],
\end{aligned}
$$

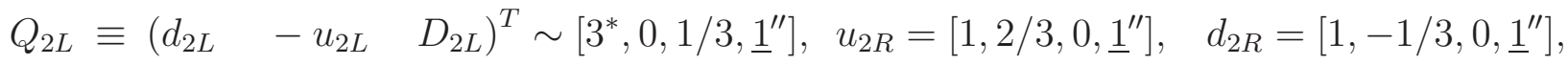

$$
\begin{aligned}
& U_{R} \sim[1,2 / 3,-1, \underline{1}], \quad D_{1 R} \sim\left[1,-1 / 3,1, \underline{1}^{\prime}\right], \quad D_{2 R} \sim\left[1,-1 / 3,1, \underline{1}^{\prime \prime}\right] .
\end{aligned}
$$

Note that the $\underline{1}, \underline{1}^{\prime}$ and $\underline{1}^{\prime \prime}$ for quarks meets the requirement of the anomaly cancellation condition in the 3-3-1 models because one family of quarks transforms differently from the others. In what follows, we consider the possibilities for generating the quark masses. The scalar multiplets needed for this purpose will be introduced accordingly.

To generate masses for the charged leptons, we introduce two $S U(3)_{L}$ scalar triplets $\phi$ and $\phi^{\prime}$, respectively, lying in $\underline{1}$ and $\underline{1}^{\prime \prime \prime}$ under $D_{4}$, with the $\operatorname{VEVs}\langle\phi\rangle=\left(\begin{array}{lll}0 & v & 0\end{array}\right)^{T}$ and $\left\langle\phi^{\prime}\right\rangle=\left(\begin{array}{lll}0 & v^{\prime} & 0\end{array}\right)^{T}[23]$. From the Yukawa interactions for the charged leptons, we get $m_{e}=h_{1} v, m_{\mu}=h v-h^{\prime} v^{\prime}$ and $m_{\tau}=h v+h^{\prime} v^{\prime}$, and the left- and the right-handed charged leptons mixing matrices are obtained [28]

$$
U_{l}=U_{R} \simeq\left(\begin{array}{ccc}
1 & 0 & 0 \\
0 & \frac{1}{\sqrt{2}} & \frac{1}{\sqrt{2}} \\
0 & -\frac{1}{\sqrt{2}} & \frac{1}{\sqrt{2}}
\end{array}\right)
$$

In similarity to the charged lepton sector, to generate the neutrino masses, we have additionally introduced the two scalar Higgs anti-sextets $s, \sigma$, respectively, lying in $\underline{1}, \underline{1}$ and $\underline{1}^{\prime}$ under $D_{4}$, and one $\mathrm{SU}(3)_{L}$ triplet lying in $\underline{1}^{\prime \prime \prime}$ under $D_{4}$, whose contribution is regarded as a small perturbation. The neutrino mass and mixing are then consistent with the experimental data

given in Ref. 16 in both normal and inverted hierarchical patterns. For a detailed study on the charged-lepton and neutrino sectors, the reader is referred to Ref. 28. 


\section{QUARK MASS AND MIXING}

\section{A. The Tree Level}

Let's us recall the two $S U(3)_{L}$ Higgs scalars responsible for charged lepton masses [28]:

$$
\phi=\left(\phi_{1}^{+} \phi_{2}^{0} \phi_{3}^{+}\right)^{T} \sim[3,2 / 3,-1 / 3, \underline{1}], \quad \phi^{\prime}=\left(\phi_{1}^{\prime+} \phi_{2}^{\prime 0} \phi_{3}^{\prime+}\right)^{T} \sim\left[3,2 / 3,-1 / 3, \underline{1}^{\prime \prime \prime}\right] .
$$

To generate the mass for quarks with a minimal Higgs content, we introduce following $S U(3)_{L}$ Higgs triplets:

$$
\begin{aligned}
\chi & =\left(\begin{array}{lll}
\chi_{1}^{0} & \chi_{2}^{-} & \chi_{3}^{0}
\end{array}\right)^{T} \sim[3,-1 / 3,2 / 3, \underline{1}], \\
\eta & =\left(\begin{array}{lll}
\eta_{1}^{0} & \eta_{2}^{-} & \eta_{3}^{0}
\end{array}\right)^{T} \sim[3,-1 / 3,-1 / 3, \underline{1}], \\
\eta^{\prime} & =\left(\begin{array}{lll}
\eta_{1}^{\prime 0} & \eta_{2}^{\prime-} & \eta_{3}^{\prime 0}
\end{array}\right)^{T} \sim\left[3,-1 / 3,-1 / 3, \underline{1}^{\prime \prime \prime}\right] .
\end{aligned}
$$

The Yukawa interactions are

$$
\begin{aligned}
-\mathcal{L}_{q} & =h_{3}^{d} \bar{Q}_{3 L} \phi d_{3 R}+h_{1}^{u} \bar{Q}_{1 L} \phi^{*} u_{1 R}+h_{2}^{u} \bar{Q}_{2 L} \phi^{*} u_{2 R}+h^{\prime u}\left(\bar{Q}_{1 L} u_{2 R}+\bar{Q}_{2 L} u_{1 R}\right) \phi^{\prime *} \\
& +h_{3}^{u} \bar{Q}_{3 L} \eta u_{3 R}+h_{1}^{d} \bar{Q}_{1 L} \eta^{*} d_{1 R}+h_{2}^{d} \bar{Q}_{2 L} \eta^{*} d_{2 R}+h^{\prime d}\left(\bar{Q}_{1 L} d_{2 R}+\bar{Q}_{2 L} d_{1 R}\right) \eta^{*} \\
& +f_{1} \bar{Q}_{1 L} \chi^{*} D_{1 R}+f_{2} \bar{Q}_{2 L} \chi^{*} D_{2 R}+f_{3} \bar{Q}_{3 L} \chi U_{R}+\text { H.C. }
\end{aligned}
$$

We should mention that the VEVs of $\chi, \phi, \eta$ conserve $D_{4}$ while those of $\phi^{\prime}, \eta^{\prime}$ break this symmetry into $Z_{2} \otimes Z_{2}$ [28]. Therefore, in the quark sector, $D_{4}$ group is broken into $Z_{2} \otimes Z_{2}$. We assume that the $\mathrm{VEVs}$ of $\chi, \phi, \phi^{\prime}, \eta, \eta^{\prime}$, respectively, are given as

$$
\begin{aligned}
& \langle\chi\rangle=\left(\begin{array}{lll}
0 & 0 & v_{\chi}
\end{array}\right)^{T}, \\
& \langle\phi\rangle=\left(\begin{array}{lll}
0 & v & 0
\end{array}\right)^{T}, \quad\left\langle\phi^{\prime}\right\rangle=\left(\begin{array}{lll}
0 & v^{\prime} & 0
\end{array}\right)^{T} \\
& \langle\eta\rangle=\left(\begin{array}{llll}
u & 0 & 0
\end{array}\right)^{T}, \quad\left\langle\eta^{\prime}\right\rangle=\left(\begin{array}{llll}
u^{\prime} & 0 & 0
\end{array}\right)^{T}
\end{aligned}
$$

The mass Lagrangian for quarks is then given by

$$
\begin{aligned}
-\mathcal{L}_{q}^{\text {mass }} & =h_{3}^{d} v \bar{d}_{3 L} d_{3 R}-h_{1}^{u} v^{*} \bar{u}_{1 L} u_{1 R}-h_{2}^{u} v^{*} \bar{u}_{2 L} u_{2 R}-h^{\prime u} v^{\prime *}\left(\bar{u}_{1 L} u_{2 R}+\bar{u}_{2 L} u_{1 R}\right) \\
& +h_{3}^{u} u \bar{u}_{3 L} u_{3 R}+h_{1}^{d} u^{*} \bar{d}_{1 L} d_{1 R}+h_{2}^{d} u^{*} \bar{d}_{2 L} d_{2 R}+h^{\prime d} u^{\prime *}\left(\bar{d}_{1 L} d_{2 R}+\bar{d}_{2 L} d_{1 R}\right) \\
& +f_{3} v_{\chi} \bar{U}_{L} U_{R}+f_{1} v_{\chi}^{*} \bar{D}_{1 L} D_{1 R}+f_{2} v_{\chi}^{*} \bar{D}_{2 L} D_{2 R}+\text { H.C. }
\end{aligned}
$$

The exotic quarks get masses

$$
m_{U}=f_{3} v_{\chi}, \quad m_{D_{1,2}}=f_{1,2} v_{\chi}
$$


The mass matrices for ordinary up- and down-quarks are, respectively, obtained as follows:

$$
M_{u}=\left(\begin{array}{ccc}
-h_{1}^{u} v & -h^{\prime u} v^{\prime} & 0 \\
-h^{\prime u} v^{\prime} & -h_{2}^{u} v & 0 \\
0 & 0 & h_{3}^{u} u
\end{array}\right), M_{d}=\left(\begin{array}{ccc}
h_{1}^{d} u & h^{\prime d} u^{\prime} & 0 \\
h^{\prime d} u^{\prime} & h_{2}^{d} u & 0 \\
0 & 0 & h_{3}^{d} v
\end{array}\right) .
$$

The matrices $M_{u}, M_{d}$ in (12) are diagonalized as

$$
\begin{aligned}
V_{L}^{u+} M_{u} V_{R}^{u} & =\operatorname{diag}\left(m_{u}, m_{c}, m_{t}\right), \\
V_{L}^{d+} M_{d} V_{R}^{d} & =\operatorname{diag}\left(m_{d}, m_{s}, m_{b}\right),
\end{aligned}
$$

where

$$
\begin{aligned}
& m_{u}=-\frac{1}{2}\left[\left(h_{1}^{u}+h_{2}^{u}\right) v+\sqrt{\left(h_{1}^{u}-h_{2}^{u}\right)^{2} v^{2}+\left(2 h^{\prime u} v^{\prime}\right)^{2}}\right], \\
& m_{c}=-\frac{1}{2}\left[\left(h_{1}^{u}+h_{2}^{u}\right) v-\sqrt{\left(h_{1}^{u}-h_{2}^{u}\right)^{2} v^{2}+\left(2 h^{\prime u} v^{\prime}\right)^{2}}\right], \quad m_{t}=h_{3}^{u} u, \\
& m_{d}=\frac{1}{2}\left[\left(h_{1}^{d}+h_{2}^{d}\right) u-\sqrt{\left(h_{1}^{d}-h_{2}^{d}\right)^{2} u^{2}+\left(2 h^{\prime d} u^{\prime}\right)^{2}}\right], \\
& m_{s}=\frac{1}{2}\left[\left(h_{1}^{d}+h_{2}^{d}\right) u+\sqrt{\left(h_{1}^{d}-h_{2}^{d}\right)^{2} u^{2}+\left(2 h^{\prime d} u^{\prime}\right)^{2}}\right], \quad m_{b}=h_{3}^{d} v .
\end{aligned}
$$

and

$$
U_{L}^{u}=U_{R}^{u}=\left(\begin{array}{ccc}
\frac{K}{\sqrt{K^{2}+1}} & -\frac{1}{\sqrt{K^{2}+1}} & 0 \\
\frac{1}{\sqrt{K^{2}+1}} & \frac{K}{\sqrt{K^{2}+1}} & 0 \\
0 & 0 & 1
\end{array}\right), \quad U_{L}^{d}=U_{R}^{d}=\left(\begin{array}{ccc}
\frac{A}{\sqrt{A^{2}+1}} & -\frac{1}{\sqrt{A^{2}+1}} & 0 \\
\frac{1}{\sqrt{A^{2}+1}} & \frac{A}{\sqrt{A^{2}+1}} & 0 \\
0 & 0 & 1
\end{array}\right)
$$

with

$$
\begin{aligned}
& K=\frac{\left(h_{1}^{u}-h_{2}^{u}\right) v+\sqrt{\left(h_{1}^{u}-h_{2}^{u}\right)^{2} v^{2}+\left(2 h^{\prime u} v^{\prime}\right)^{2}}}{2 h^{\prime u} v^{\prime}} \\
& A=\frac{\left(h_{1}^{d}-h_{2}^{d}\right) u-\sqrt{\left(h_{1}^{d}-h_{2}^{d}\right)^{2} u^{2}+\left(2 h^{\prime d} u^{\prime}\right)^{2}}}{2 h^{\prime d} u^{\prime}} .
\end{aligned}
$$

The CKM matrix is defined as

$$
U_{C K M}=U_{L}^{u} U_{L}^{d+}=\left(\begin{array}{ccc}
\frac{1+A K}{\sqrt{A^{2}+1} \sqrt{K^{2}+1}} & \frac{K-A}{\sqrt{A^{2}+1} \sqrt{K^{2}+1}} & 0 \\
\frac{A-K}{\sqrt{A^{2}+1} \sqrt{K^{2}+1}} & \frac{1+A K}{\sqrt{A^{2}+1} \sqrt{K^{2}+1}} & 0 \\
0 & 0 & 1
\end{array}\right)
$$

where $K$ and $A$ are defined in Eqs. (18) and (19). In the special case $K=A$, i.e,

$$
\frac{u}{u^{\prime}}=\frac{h_{1}^{u}-h_{2}^{u}}{h_{1}^{d}-h_{2}^{d}} \frac{h^{\prime d}}{h^{\prime}} \frac{v}{v^{\prime}},
$$


the $U_{C K M}$ in Eq.(20) reduces to the identity.

In the model under consideration, the following limit is often taken into account [2123, 32, 33]:

$$
u \sim u^{\prime} \sim v^{\prime} \sim v
$$

On the other hand, taking into account the discovery of the long-awaited Higgs boson at around $125 \mathrm{GeV}$ by ATLAS [34] and CMS [35], we can estimate the VEVs as follows:

$$
u \sim u^{\prime} \sim v^{\prime} \sim v=100 \mathrm{GeV}
$$

The matrix $U_{C K M}$ in Eq. (20) is closer to the realistic quark mixing matrix than those derived at the tree level from other discrete symmetry groups [21 23, 25 29]. Indeed, with the help of Eq. (22) and by taking the experimental data on quark mass [16]

$$
\begin{aligned}
& m_{u}=2.3 \mathrm{MeV}, \quad m_{c}=1.275 \mathrm{GeV}, \quad m_{t}=173.21 \mathrm{GeV}, \\
& m_{d}=4.8 \mathrm{MeV}, \quad m_{s}=95 \mathrm{MeV}, \quad m_{b}=4.18 \mathrm{GeV},
\end{aligned}
$$

as well as the average values of the CKM matrix elements in Ref.[16] given in Eq.(11). With $\left|U_{u d}\right|=0.97425 \pm 0.00022$, we get solutions for $A, K$ and the Yukawa quark couplings

$h_{1,2,3}^{u}, h^{\prime u}, h_{1,2,3}^{d}, h^{\prime d}$ which are listed in table \. We see that the matrix in table \ is close to the realistic quark mixing matrix; i.e, the deviations of the matrix $U_{C K M}$ in Eq.(1) from the matrix in table $\amalg$ are very small, so this is a good approximation for the realistic quark mixing matrix, which implies that the mixings among the quarks are dynamically small. This is one of the most striking predictions of the model under consideration. As we will see in section IIIB, a violation of $D_{4}$ symmetry due to Yukawa interactions will disturb the tree-level matrix, resulting in mixing between ordinary quarks and providing the desirable quark mixing pattern.

\section{B. The First-Order Corrections}

All terms of the Yukawa interactions responsible for the quarks masses in Eq. (6) are invariant under the $\left[\mathrm{SU}(3)_{L}, \mathrm{U}(1)_{X}, \mathrm{U}(1)_{\mathcal{L}}, \underline{D}_{4}\right]$ symmetries. To obtain a realistic quark mixing, here we add some terms violating $D_{4}$ symmetry with $1^{\prime}$. These terms are 
TABLE I: Model parameters derived from the fit with the data in Ref. [16] at the tree - level .

\begin{tabular}{|c|c|c|}
\hline$A, K$ & $h_{1}^{u}, h_{2}^{u}, h_{3}^{u}, h^{\prime u}$ & $U_{C K M}$ \\
\hline $0.65558,1.04565$ & $\begin{array}{c}-0.00610,-0.00667,1.73500,0.00636 \\
0.000319,0.00068,0.04180,0.00041\end{array}$ & $\begin{array}{ccc}0.97425 & 0.22547 & 0 \\
-0.22547 & 0.97425 & 0 \\
0 & 0 & 1\end{array}$ \\
\hline
\end{tabular}

$\widetilde{Q}_{1 L} \phi^{*} u_{3 R}, \widetilde{Q}_{1 L} \eta^{*} d_{3 R}, \widetilde{Q}_{3 L} \eta u_{1 R}$ and $\widetilde{Q}_{3 L} \phi d_{1 R}$. Hence, the total Yukawa couplings of the ordinary quarks have two extra terms $-\Delta \mathcal{L}_{q}^{u}$ and $-\Delta \mathcal{L}_{q}^{d}$ which are given by

$$
\begin{aligned}
-\Delta \mathcal{L}_{q}^{u} & =k_{1}^{u} \widetilde{Q}_{1 L} \phi^{*} u_{3 R}+k_{2}^{u} \widetilde{Q}_{3 L} \eta u_{1 R}+H . C \\
-\Delta \mathcal{L}_{q}^{d} & =k_{1}^{d} \widetilde{Q}_{1 L} \eta^{*} d_{3 R}+k_{2}^{d} \widetilde{Q}_{3 L} \phi d_{1 R}+H . C .
\end{aligned}
$$

The total mass matrices for the ordinary up-quarks and down-quarks then take the forms:

$$
M_{u}^{\prime}=\left(\begin{array}{ccc}
-h_{1}^{u} v & -h^{\prime u} v^{\prime} & -k_{1}^{u} v \\
-h^{\prime u} v^{\prime} v & -h_{2}^{u} v & 0 \\
k_{2}^{u} u & 0 & h_{3}^{u} u
\end{array}\right), M_{d}^{\prime}=\left(\begin{array}{ccc}
h_{1}^{d} u & h^{\prime d} u^{\prime} & k_{1}^{d} u \\
h^{\prime d} u^{\prime} & h_{2}^{d} u & 0 \\
k_{2}^{d} v & 0 & h_{3}^{d} v
\end{array}\right) .
$$

We can separate the quark mass matrices in Eq.(26) into two parts as follows:

$$
M_{u}^{\prime}=M_{u}+\Delta M_{u}, \quad M_{d}^{\prime}=M_{d}+\Delta M_{d}
$$

where $M_{u}$ and $M_{d}$ are given by Eq. (12) due to the contributions of the invariant terms only, and

$$
\Delta M_{u}=\left(\begin{array}{ccc}
0 & 0 & -k_{1}^{u} v \\
0 & 0 & 0 \\
k_{2}^{u} u & 0 & 0
\end{array}\right), \Delta M_{d}=\left(\begin{array}{ccc}
0 & 0 & k_{1}^{d} u \\
0 & 0 & 0 \\
k_{2}^{d} v & 0 & 0
\end{array}\right)
$$

are deviations from the contributions of the $D_{4}$ violation terms. In the case without $D_{4}$ violation, the first terms can approximately fit the data in Ref. 16 with very small deviations, as was shown in section IIIA. The second terms belong to the contributions of the $D_{4}$ violation in Eqs. (24) and (25). Then, we can consider the contributions of $D_{4}$ violation as small perturbations in the quark sector and terminate the theory at the first order. At this approximation, the matrices $\Delta M_{u}$ and $\Delta M_{d}$ in Eq. (28) do not contribute to the 
quark eigenvalues. However, they change the corresponding eigenvectors. The up- and the down-quark masses are, thus, obtained as

$$
m_{i}^{\prime}=m_{i} \quad(i=u, c, t, d, s, b),
$$

where $m_{i}(i=u, c, t, d, s, b)$ are given in Eqs.(15) and (16).

The unitary matrices that couple the left-handed quarks $u_{L}$ and $d_{L}$ to those in the mass bases, respectively, are

$$
\begin{aligned}
U_{L}^{\prime u}=\left(\begin{array}{ccc}
\frac{K}{\sqrt{K^{2}+1}} & -\frac{1}{\sqrt{K^{2}+1}} & \frac{k_{1}^{u} v\left[K^{2}\left(m_{c}-m_{t}\right)+\left(m_{u}-m_{t}\right)\right]}{\left(K^{2}+1\right)\left(m_{u}-m_{t}\right)\left(m_{c}-m_{t}\right)} \\
\frac{1}{\sqrt{K^{2}+1}} & \frac{K}{\sqrt{K^{2}+1}} & -\frac{K k_{1}^{u} v\left(m_{u}-m_{c}\right)}{\left(K^{2}+1\right)\left(m_{u}-m_{t}\right)\left(m_{c}-m_{t}\right)} \\
\frac{K k_{2}^{u} u}{\sqrt{K^{2}+1}\left(m_{u}-m_{t}\right)} & -\frac{k_{2}^{u} u}{\sqrt{K^{2}+1}\left(m_{c}-m_{t}\right)} & 1
\end{array}\right), \\
U_{L}^{\prime d}=\left(\begin{array}{ccc}
\frac{A}{\sqrt{A^{2}+1}} & -\frac{1}{\sqrt{A^{2}+1}} & -\frac{k_{1}^{d} u\left[A^{2}\left(m_{s}-m_{b}\right)+\left(m_{d}-m_{b}\right)\right]}{\left(A^{2}+1\right)\left(m_{d}-m_{b}\right)\left(m_{s}-m_{b}\right)} \\
\frac{1}{\sqrt{A^{2}+1}} & \frac{A}{\sqrt{A^{2}+1}} & \frac{A k_{1}^{d} u\left(m_{d}-m_{s}\right)}{\left(A^{2}+1\right)\left(m_{d}-m_{b}\right)\left(m_{s}-m_{b}\right)} \\
\frac{A k_{2}^{d} v}{\sqrt{A^{2}+1}\left(m_{d}-m_{b}\right)} & -\frac{k_{2}^{d} v}{\sqrt{A^{2}+1}\left(m_{s}-m_{b}\right)} & 1
\end{array}\right),
\end{aligned}
$$

where $A, K$ are given in Eqs. (19) and (18). The CKM matrix at the first order of perturbation theory is now defined as [16]

$$
U_{C K M}^{\prime}=U_{L}^{\prime u} U_{L}^{\prime d+}=\left(\begin{array}{ccc}
U_{11} & U_{12} & U_{13} \\
U_{21} & U_{22} & U_{23} \\
U_{31} & U_{32} & U_{33}
\end{array}\right),
$$

where $U_{i j}(i, j=1,2,3)$ are given in appendix $\mathrm{B}$.

Our model is easily shown to be consistent because the experimental constraints on the mixing angles and the masses of quarks can be, respectively, fitted with the quark Yukawa coupling parameters $h_{1,2,3}^{u}, h_{1,2,3}^{d}, h^{\prime u}, h^{\prime d}, k_{1,2}^{u}, k_{1,2}^{d}$ of all the $S U(3)_{L}$ triplet scalars, provided that the VEVs $u, u^{\prime}, v, v^{\prime}$ and the quark masses are given by Eqs. (22) and (23), respectively. Indeed, by comparing the elements of $U_{C K M}^{\prime}$ in Eq. (32) with the corresponding best fit values given in Ref. 16, we get a solution $A=-K=0.114156$, a prediction for quark mixing as presented in table II, and

$$
\begin{aligned}
& h_{1}^{u}=-1.2586 \times 10^{-2}, h_{2}^{u}=-1.8672 \times 10^{-4}, h_{3}^{u}=1.735, h^{\prime u}=1.43417 \times 10^{-3},(33) \\
& h_{1}^{d}=7.37265 \times 10^{-4}, h_{2}^{d}=2.60736 \times 10^{-4}, h_{3}^{d}=4.18 \times 10^{-2}, h^{\prime d}=3.82925 \times 10^{-4} .
\end{aligned}
$$

The results in Eq. (33) and table II show that $h_{1}^{u}, h_{2}^{u}, h^{\prime u} \ll h_{3}^{u}$ and $h_{1}^{d}, h_{2}^{d}, h^{\prime d} \ll h_{3}^{d}$. There is a consequence of the fact that the top- and the bottom-quark masses are much larger than those of the others. 
TABLE II: Elements of the quark mixing matrix from the model at the first order

\begin{tabular}{ccc}
\hline \hline Elements & The prediction & The best fit fom Ref. 16 \\
\hline$U_{u d}$ & 0.94347 & 0.97425 \\
\hline$U_{u s}$ & 0.2253 & 0.2253 \\
\hline$U_{u b}$ & 0.00413 & 0.00413 \\
\hline$U_{c d}$ & 0.2254 & 0.225 \\
\hline$U_{c s}$ & 0.9743 & 0.986 \\
\hline$U_{c b}$ & 0.0411 & 0.0411 \\
\hline$U_{t d}$ & 0.0084 & 0.0084 \\
\hline$U_{t s}$ & 0.04001 & 0.040 \\
\hline$U_{t b}$ & 0.96753 & 1.021 \\
\hline \hline
\end{tabular}

\section{CONCLUSION}

In this paper, we have proposed a new $D_{4}$ flavor model based on $\mathrm{SU}(3)_{C} \otimes \mathrm{SU}(3)_{L} \otimes \mathrm{U}(1)_{X}$ gauge symmetry in which the quark mixing matrix is concentrated. After spontaneous breaking of flavor symmetry, with a constraint on Higgs VEVs in the Yukawa couplings, all of quarks have consistent masses and a realistic quark mixing matrix can be realized at the first order of perturbation theory. Numerical estimation shows that the Yukawa couplings in the model under consideration are consistent with those in the SM.

\section{ACKNOWLEDGMENTS}

This research is funded by the Vietnam National Foundation for Science and Technology Development (NAFOSTED) under grant number 103.01-2014.51.

\section{Appendix A: $D_{4}$ group and Clebsch-Gordan coefficients}

$D_{4}$ is the symmetry group of a square. It has eight elements divided into five conjugacy classes, with $\underline{1}, \underline{1}^{\prime}, \underline{1}^{\prime \prime}, \underline{1}^{\prime \prime \prime}$ and $\underline{2}$ as its five irreducible representations. Any element of $D_{4}$ can be formed by multiplication of the generators $a$ (the $\pi / 2$ rotation) and $b$ (the reflection) obeying the relations $a^{4}=e, b^{2}=e$, and $b a b=a^{-1}$. $D_{4}$ has the following five conjugacy 
classes,

$$
\begin{aligned}
& C_{1}:\left\{a_{1} \equiv e\right\}, \\
& C_{2}:\left\{a_{2} \equiv a^{2}\right\}, \\
& C_{3}:\left\{a_{3} \equiv a, a_{4} \equiv a^{3}\right\}, \\
& C_{4}:\left\{a_{5} \equiv b, a_{6} \equiv a^{2} b\right\}, \\
& C_{5}:\left\{a_{7} \equiv a b, a_{8} \equiv a^{3} b\right\} .
\end{aligned}
$$

The character table of $D_{4}$ is given in table III, where $n$ is the order of class and $h$ is the order of elements within each class.

TABLE III: The character table of $D_{4}$

\begin{tabular}{llllcccc}
\hline \hline Class & $n$ & $h$ & $\chi_{1}$ & $\chi_{1^{\prime}}$ & $\chi_{1^{\prime \prime}}$ & $\chi_{1^{\prime \prime \prime}}$ & $\chi_{2}$ \\
\hline$C_{1}$ & 1 & 1 & 1 & 1 & 1 & 1 & 2 \\
$C_{2}$ & 1 & 2 & 1 & 1 & 1 & 1 & -2 \\
$C_{3}$ & 2 & 4 & 1 & -1 & -1 & 1 & 0 \\
$C_{4}$ & 2 & 2 & 1 & 1 & -1 & -1 & 0 \\
$C_{5}$ & 2 & 2 & 1 & -1 & 1 & -1 & 0 \\
\hline \hline
\end{tabular}

We have worked in a real basis, in which the two-dimensional representation $\underline{2}$ of $D_{4}$ is real, $2^{*}\left(1^{*}, 2^{*}\right)=2\left(1^{*}, 2^{*}\right)$. One possible choice of generators is given as

$$
\begin{aligned}
& \underline{1}: a=1, \quad b=1, \\
& \underline{1}^{\prime}: a=1, \quad b=-1, \\
& \underline{1}^{\prime \prime}: a=-1, \quad b=1, \\
& \underline{1}^{\prime \prime \prime}: a=-1, \quad b=-1, \\
& \underline{2}: a=\left(\begin{array}{cc}
0 & 1 \\
-1 & 0
\end{array}\right), \quad b=\left(\begin{array}{cc}
1 & 0 \\
0 & -1
\end{array}\right) .
\end{aligned}
$$

Using them, we calculate the Clebsch-Gordan coefficients for all the tensor products as given below.

First, let us put $\underline{2}(1,2)$, which means some $\underline{2}$ doublet such as $x=\left(x_{1}, x_{2}\right) \sim \underline{2}$ or $y=$ $\left(y_{1}, y_{2}\right) \sim \underline{2}$ and so on, and similarly for the other representations. Moreover, the numbered 
multiplets such as $(\ldots, i j, \ldots)$ mean $\left(\ldots, x_{i} y_{j}, \ldots\right)$, where $x_{i}$ and $y_{j}$ are the multiplet components of different representations $x$ and $y$, respectively. In the following, the components of the representations on the left-hand sides will be omitted and should be understood, but they always exist in order in the components of the decompositions on the right-hand sides:

$$
\begin{aligned}
\underline{1}(1) \otimes \underline{1}(1) & =\underline{1}(11), \quad \underline{1}^{\prime}(1) \otimes \underline{1}^{\prime}(1)=\underline{1}(11), \\
\underline{1}^{\prime \prime}(1) \otimes \underline{1}^{\prime \prime}(1) & =\underline{1}(11), \quad \underline{1}^{\prime \prime \prime}(1) \otimes \underline{1}^{\prime \prime \prime}(1)=\underline{1}(11), \\
\underline{1}(1) \otimes \underline{1}^{\prime}(1) & =\underline{1}^{\prime}(11), \quad \underline{1}(1) \otimes \underline{1}^{\prime \prime}(1)=\underline{1}^{\prime \prime}(11), \\
\underline{1}(1) \otimes \underline{1}^{\prime \prime \prime}(1) & =\underline{1}^{\prime \prime \prime}(11), \quad \underline{1}^{\prime}(1) \otimes \underline{1}^{\prime \prime}(1)=\underline{1}^{\prime \prime \prime}(11), \\
\underline{1}^{\prime \prime}(1) \otimes \underline{1}^{\prime \prime \prime}(1) & =\underline{1}^{\prime}(11), \quad \underline{1}^{\prime \prime \prime}(1) \otimes \underline{1}^{\prime}(1)=\underline{1}^{\prime \prime}(11), \\
\underline{1}(1) \otimes \underline{2}(1,2) & =\underline{2}(11,12), \quad \underline{1}^{\prime}(1) \otimes \underline{2}(1,2)=\underline{2}(11,-12), \\
\underline{1}^{\prime \prime}(1) \otimes \underline{2}(1,2) & =\underline{2}(12,11), \quad \underline{1}^{\prime \prime \prime}(1) \otimes \underline{2}(1,2)=\underline{2}(-12,11), \\
\underline{2}(1,2) \otimes \underline{2}(1,2) & =\underline{1}(11+22) \oplus \underline{1}^{\prime}(11-22) \oplus \underline{1}^{\prime \prime}(12+21) \oplus \underline{1}^{\prime \prime \prime}(12-21) .
\end{aligned}
$$

In the text, we usually use the following notations, for example, $(x y)_{1} \equiv\left(x_{1} y_{1}+x_{2} y_{2}\right)$, which is the Clebsch-Gordan coefficients of $\underline{1}$ in the decomposition of $\underline{2} \otimes \underline{2}$, where as mentioned $x=\left(x_{1}, x_{2}\right) \sim \underline{2}$ and $y=\left(y_{1}, y_{2}\right) \sim \underline{2}$. The rules to conjugate the representations $\underline{1}$, $\underline{1}^{\prime}, \underline{1}^{\prime \prime}, \underline{1}^{\prime \prime \prime}$ and $\underline{2}$ are given by

$$
\begin{aligned}
\underline{2}^{*}\left(1^{*}, 2^{*}\right) & =\underline{2}\left(1^{*}, 2^{*}\right), \\
\underline{1}^{*}\left(1^{*}\right) & =\underline{1}\left(1^{*}\right), \underline{1}^{* *}\left(1^{*}\right)=\underline{1}^{\prime}\left(1^{*}\right), \underline{1}^{\prime \prime *}\left(1^{*}\right)=\underline{1}^{\prime \prime}\left(1^{*}\right), \underline{1}^{\prime \prime \prime *}\left(1^{*}\right)=\underline{1}^{\prime \prime \prime}\left(1^{*}\right),
\end{aligned}
$$

where, for example, $\underline{2}^{*}\left(1^{*}, 2^{*}\right)$ denotes some $\underline{2}^{*}$ multiplet of the form $\left(x_{1}^{*}, x_{2}^{*}\right) \sim \underline{2}^{*}$. 


\section{Appendix B: Elements of the matrix $U_{C K M}^{\prime}$}

$$
\begin{aligned}
& U_{11}=\frac{A K+1}{\sqrt{A^{2}+1} \sqrt{K^{2}+1}}-\frac{\left[K^{2}\left(m_{c}-m_{t}\right)+\left(m_{u}-m_{t}\right)\right]\left[A^{2}\left(m_{s}-m_{b}\right)+\left(m_{d}-m_{b}\right)\right] k_{1}^{u} k_{1}^{d} u v}{\left(A^{2}+1\right)\left(K^{2}+1\right)\left(m_{u}-m_{t}\right)\left(m_{c}-m_{t}\right)\left(m_{s}-m_{b}\right)\left(m_{d}-m_{b}\right)}, \\
& U_{12}=\frac{K-A}{\sqrt{A^{2}+1} \sqrt{K^{2}+1}}+\frac{A\left[K^{2}\left(m_{c}-m_{t}\right)+\left(m_{u}-m_{t}\right)\right]\left(m_{d}-m_{s}\right) k_{1}^{u} k_{1}^{d} u v}{\left(A^{2}+1\right)\left(K^{2}+1\right)\left(m_{u}-m_{t}\right)\left(m_{c}-m_{t}\right)\left(m_{s}-m_{b}\right)\left(m_{d}-m_{b}\right)}, \\
& U_{13}=\frac{\left[K^{2}\left(m_{c}-m_{t}\right)+\left(m_{u}-m_{t}\right)\right] k_{1}^{u} v}{\left(K^{2}+1\right)\left(m_{u}-m_{t}\right)\left(m_{c}-m_{t}\right)}+\frac{k_{2}^{d} v}{\sqrt{A^{2}+1} \sqrt{K^{2}+1}}\left(\frac{A K}{m_{d}-m_{b}}+\frac{1}{m_{s}-m_{b}}\right) \text {, } \\
& U_{21}=\frac{A-K}{\sqrt{A^{2}+1} \sqrt{K^{2}+1}}+\frac{K\left(m_{u}-m_{c}\right)\left[A^{2}\left(m_{s}-m_{b}\right)+\left(m_{d}-m_{b}\right)\right] k_{1}^{u} k_{1}^{d} u v}{\left(A^{2}+1\right)\left(K^{2}+1\right)\left(m_{u}-m_{t}\right)\left(m_{c}-m_{t}\right)\left(m_{s}-m_{b}\right)\left(m_{d}-m_{b}\right)}, \\
& U_{22}=\frac{A K+1}{\sqrt{A^{2}+1} \sqrt{K^{2}+1}}-\frac{A K\left(m_{u}-m_{c}\right)\left(m_{d}-m_{s}\right) k_{1}^{u} k_{1}^{d} u v}{\left(A^{2}+1\right)\left(K^{2}+1\right)\left(m_{u}-m_{t}\right)\left(m_{c}-m_{t}\right)\left(m_{s}-m_{b}\right)\left(m_{d}-m_{b}\right)}, \\
& U_{23}=-\frac{K k_{1}^{u} v\left(m_{u}-m_{c}\right)}{\left(K^{2}+1\right)\left(m_{u}-m_{t}\right)\left(m_{c}-m_{t}\right)}+\frac{k_{2}^{d} v}{\sqrt{K^{2}+1} \sqrt{A^{2}+1}}\left(\frac{A}{m_{d}-m_{b}}-\frac{K}{m_{s}-m_{b}}\right) \text {, } \\
& U_{31}=-\frac{k_{1}^{d} u\left[A^{2}\left(m_{s}-m_{b}\right)+\left(m_{d}-m_{b}\right)\right]}{\left(A^{2}+1\right)\left(m_{s}-m_{b}\right)\left(m_{d}-m_{b}\right)}+\frac{k_{2}^{u} u}{\sqrt{K^{2}+1} \sqrt{A^{2}+1}}\left(\frac{A K}{m_{u}-m_{t}}+\frac{1}{m_{c}-m_{t}}\right) \text {, } \\
& U_{32}=\frac{A k_{1}^{d} u\left(m_{d}-m_{s}\right)}{\left(A^{2}+1\right)\left(m_{s}-m_{b}\right)\left(m_{d}-m_{b}\right)}+\frac{k_{2}^{u} u}{\sqrt{K^{2}+1} \sqrt{A^{2}+1}}\left(\frac{K}{m_{u}-m_{t}}-\frac{A}{m_{c}-m_{t}}\right) \text {, } \\
& U_{33}=1+\frac{k_{2}^{u} k_{2}^{d} u v}{\sqrt{K^{2}+1} \sqrt{A^{2}+1}}\left[\frac{A K}{\left(m_{u}-m_{t}\right)\left(m_{d}-m_{b}\right)}+\frac{1}{\left(m_{c}-m_{t}\right)\left(m_{s}-m_{b}\right)}\right] \text {. }
\end{aligned}
$$

\section{REFERENCES}

[1] N. Cabibbo, Phys Rev. Lett. 10, 531 (1963).

[2] M. Kobayashi and T. Maskawa, Prog. Theor. Phys. 49, 652 (1973).

[3] X-G He, S-W Li and B-Q. Ma, Phys. Rev. D 78,111301 (2008); arXiv: 0809.1223 [hep-ph].

[4] S. Antusch, S. F. King, M. Malinsky and M. Spinrath, Phys. Rev. D 81, 033008 (2010); arXiv: 0910.5127 [hep-ph].

[5] X. Zhang and B-Q Ma, Phys. Rev. D 86, 093002(2012); arXiv:1206.0519 [hep-ph].

[6] P. H. Frampton and R. N. Mohapatra, J. High Energy Phys. 0501, 025 (2005); arXiv: 0407139 [hep-ph].

[7] N. Li and B-Q. Ma, Phys. Rev. D 71, 097301 (2005); arXiv: 0501226 [hep-ph].

[8] S. K. Kang, C. S. Kim and J. Lee, Phys. Lett. B 619, 129 (2005); arXiv: 0501029 [hep-ph]. 
[9] F. Plentinger, G. Seidl and W. Winter, Phys. Rev. D 76, 113003 (2007); arXiv: 0707.2379 [hep-ph].

[10] L. Wolfenstein, Phys. Rev. Lett. 51, 1945 (1983).

[11] Y. H. Ahn, Phys. Rev. D 87, 113011 (2013); arXiv:1303.4863 [hep-ph].

[12] M. Holthausen and K.S. Lim, Phys. Rev. D 88, 033018 (2013); arXiv: 1306.4356 [hep-ph].

[13] T. Araki, H. Ishida, H. Ishimori, T. Kobayashi and A. Ogasahara, Phys. Rev. D 88, 096002 (2013); arXiv: 1309.4217 [hep-ph].

[14] A. E. Cárcamo Hernández, R Martinez and F. Ochoa, arXiv:1309.6567 [hep-ph].

[15] S. F. King, J. High Energy Phys. 01, 119 (2014); arXiv: 1311.3295 [hep-ph].

[16] Particle Data Group (K.A. Olive et al.), Chin. Phys. C 38, 090001 (2014).

[17] P. F. Harrison, D. H. Perkins and W. G. Scott, Phys. Lett. B 530, 167(2002).

[18] Z. Z. Xing, Phys. Lett. B 533, 85 (2002).

[19] X. G. He and A. Zee, Phys. Lett. B 560, 87 (2003).

[20] X. G. He and A. Zee, Phys. Rev. D 68, 037302 (2003).

[21] P. V. Dong, L. T. Hue, H. N. Long and D. V. Soa, Phys. Rev. D 81, 053004 (2010).

[22] P. V. Dong, H. N. Long, D. V. Soa, and V. V. Vien, Eur. Phys. J. C 71, 1544 (2011); arXiv:1009.2328 [hep-ph].

[23] P. V. Dong, H. N. Long, C. H. Nam, and V. V. Vien, Phys. Rev. D 85, 053001(2012); arXiv: 1111.6360 [hep-ph].

[24] V. V. Vien and H. N. Long, Int. J. Mod. Phys. A 28 (2013) 1350159; arXiv: 1312.5034 [hep-ph].

[25] V. V. Vien and H. N. Long, Adv. High Energy Phys. 2014 (2014) 192536.

[26] V. V. Vien and H. N. Long, J. Exp. Theor. Phys. 118, No. 6 (2014), 869; arXiv:1404.6119[hepph].

[27] V. V. Vien and H. N. Long, J. High Energy Phys. 04 (2014) 133; arXiv: 1402.1256 [hep-ph].

[28] V. V. Vien, Mod. Phys. Lett. A 29, No. 23 (2014) 1450122.

[29] V. V. Vien, Mod. Phys. Lett. A 29, No. 28 (2014) 1450139.

[30] Hoang Ngoc Long and Vo Van Vien, Int. J. Mod. Phys. A 29 (2014) 13, 1450072; arXiv:1405.1622 [hep-ph].

[31] Vo Van Vien, Hoang Ngoc Long and Pham Ngoc Thu, Neutrino mixing and CP violation phases in Zee-Babu model; arXiv: 1407.8286 [hep-ph]. 
[32] P. V. Dong, D. T. Huong, Tr. T. Huong and H. N. Long, Phys.Rev. D 74, 053003 (2006).

[33] P. V. Dong, H. N. Long and D. V. Soa, Phys.Rev. D 75, 073006 (2007).

[34] ATLAS Collab. (G. Aad et al.), Phys. Lett. B 716 (2012) 1; arXiv:1207.7214 [hep-ex].

[35] CMS Collab. (S. Chatrchyan et al.), Phys. Lett. B 716 (2012) 30; arXiv: 1207.7235 [hep-ex]. 